Case Report

\title{
Incidental Finding and Endovascular Repair of a Left Internal Mammary Artery Aneurysm following a Multivessel Coronary Artery Bypass Graft
}

\author{
Osayi Lawani $\mathbb{D}^{1},{ }^{1}$ Jiries Ganim $\mathbb{D}^{2},{ }^{2}$ and Rick Ganim ${ }^{3}$ \\ ${ }^{1}$ HCA Houston Healthcare-Kingwood, USA \\ ${ }^{2}$ University of Southern California, USA \\ ${ }^{3}$ Vital Heart \& Vein, USA \\ Correspondence should be addressed to Osayi Lawani; osayi.lawani@hcahealthcare.com
}

Received 21 July 2020; Revised 26 January 2021; Accepted 3 February 2021; Published 17 February 2021

Academic Editor: Filippo M. Sarullo

Copyright (C) 2021 Osayi Lawani et al. This is an open access article distributed under the Creative Commons Attribution License, which permits unrestricted use, distribution, and reproduction in any medium, provided the original work is properly cited.

\begin{abstract}
True aneurysms discovered within the internal mammary artery are extremely rare and typically have an asymptomatic occurrence. Their presentation and management have also been variable due to their low incidence, decreased detection, or lack of documentation. They have a high risk for morbidity or mortality as they can possibly rupture with increasing size and thus become life-threatening. Coronary CT angiography is the most definitive test for confirming and finding complications related to the aneurysm. With an increase in the aging population and advancement in the techniques used in coronary artery bypass grafting, it is likely that the rate of recorded occurrence of aneurysms and pseudoaneurysms will increase. Endovascular repair is currently the most favored treatment modality. In this report, we describe a case of a 74-year-old male who was incidentally found to have a left internal mammary artery aneurysm following complaints of chest pain related to another nearly occluded grafted vessel. To the best of our knowledge, and following an extensive literature review, this is likely the first documented case of a true aneurysm found within a left internal mammary artery bypass graft. The patient recovered well following placement of a covered stent; however, upon follow-up one year later, he was found to have stenosis of the same vessel, which was subsequently treated without further complication.
\end{abstract}

\section{Introduction}

Aneurysms manifesting from the internal mammary artery (IMA) are a very rare occurrence. Although they are usually small, these aneurysms will have an increased risk of rupture which can be life-threatening [1]. Other possible complications include hemothorax, thromboembolism, compression of adjacent structures, myocardial infarction, and possible death. Aneurysms in the IMA are typically found incidentally on cardiac angiography. Characteristically, these aneurysms grow slowly and silently, making this a highly underdiagnosed disease process [2].

The left IMA (LIMA) is the vessel used most to bypass a significant stenosis of the left anterior descending (LAD) artery [3]. Advantages of using the LIMA over a saphenous vein graph are long-term patency and survival rates greater than $90 \%$ beyond 10 years and decreased postoperative mortality [4]. The exact cause of an IMA aneurysm is unknown, however, it can be traumatic or iatrogenic in origin, caused by progressive atherosclerosis, or due to connective tissue disorders such as Marfan, Loeys-Dietz, and Ehlers-Danlos syndromes [5]. In this report, we present a case of an elderly male who was found to have a large aneurysm located in a LIMA to LAD artery graft following a coronary bypass procedure 6 months prior.

\section{Case History}

A 74-year-old male with a past medical history of coronary artery disease with cardiac stenting, peripheral artery disease with femoral popliteal bypass, hypertension, hyperlipidemia, diabetes mellitus, chronic kidney disease, deep vein 
thrombosis, and distant history of heavy tobacco use, presented to a local emergency department with complaints of symptoms that were suggestive of unstable angina. Cardiology was consulted and a left heart catheterization was performed.

The left main had mild diffuse plaquing. The LAD was of large caliber, and in the midsection at the takeoff of a large diagonal branch, there were stents in the artery and in the ostium of the diagonal branch, which had about $60 \%$ and $90 \%$ in-stent restenosis, respectively. The left circumflex artery was small in caliber, and in the proximal section there was $85 \%$ stenosis. The ostium of the first obtuse marginal branch had $70 \%$ stenosis. The right coronary artery was occluded in its midsegment, and the distal coronary system was able to perfuse left-to-right collateral circulation. Finally, the left internal mammary artery was widely patent. Given the multivessel coronary artery disease and small caliber of the left circumflex artery, it was recommended that the patient be evaluated by cardiothoracic surgery for a coronary artery bypass graft (CABG).

Six months later, the patient presented to his cardiologist with complaints of intermittent episodes of nitroglycerinresponsive, substernal chest pain that radiated down both arms with strenuous activity. The patient had undergone a six-vessel CABG five months earlier without any residual complications to date. Home medications included dual antiplatelet therapy, antihypertensives, antianginal agents, and a lipid-lowering agent. An electrocardiogram showed normal sinus rhythm, occasional premature atrial contractions, and no ischemic changes. Laboratory results showed elevated troponin I. He was subsequently admitted to the hosptial for cardiac catheterization.

The distal left main had $40-50 \%$ stenosis just proximal to the bifurcation of the LAD and left circumflex arteries. The proximal-to-mid portion of the LAD continued to have $60 \%$ in-stent restenosis. The ostium of the diagonal branch also continued to have $90 \%$ in-stent restenosis. The proximal portion of the left circumflex had increased stenosis from $85 \%$ to $99 \%$, and the midsegment was now occluded. The right coronary artery remained occluded in its midsegment with collateral circulation. A saphenous vein graft to the right coronary artery had $50 \%$ stenosis. A saphenous vein graft to the obtuse marginal branch in the mid-to-distal segment had 95\% stenosis. A LIMA graph to the LAD was patent; however, there was a large aneurysm within the LIMA with TIMI-3 blood flow (Figure 1). The culprit vessel causing the intermittent episodes of chest pain was felt to be the saphenous vein graph to the obtuse marginal branch, and a drugeluting stent was placed.

As for the large aneurysm found in the LIMA, it was decided that it would either be treated by surgical repair or by percutaneous intervention at a later date. The patient was discharged home to continue dual antiplatelet therapy and start aggressive blood pressure and lipid control. Three weeks later, the patient returned to the hospital to undergo repair of the large aneurysm found in the LIMA to LAD bypass graft. Following multiple discussions with and evaluation by cardiothoracic surgery, it was concluded that the patient would best benefit from placing a covered stent to

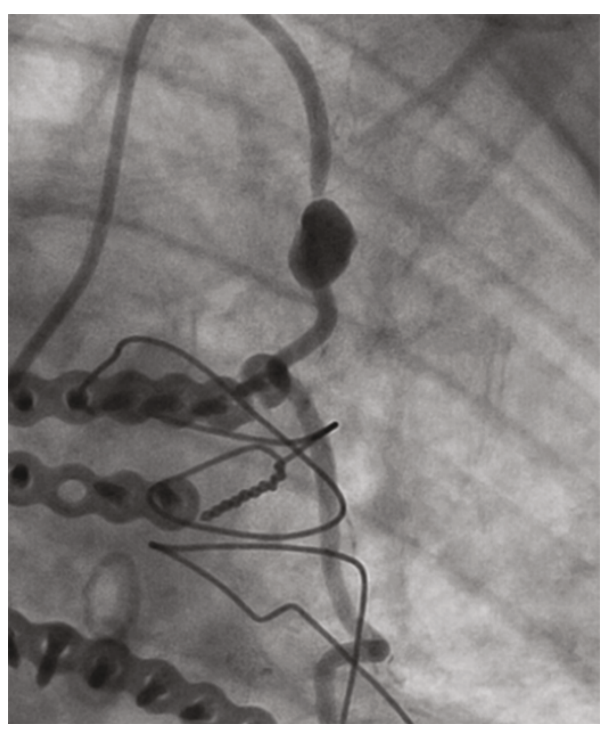

FIGURE 1: Large aneurysm in the left internal mammary artery to the left anterior descending artery bypass graft.

repair the aneurysm. Intravascular ultrasound was used to determine the size and length of the lesion. A $2.8 \times 19 \mathrm{~mm}$ covered stent was placed in the midsegment of the aneurysm (Figure 2). Postintervention angiography showed TIMI-3 blood flow with no residual aneurysm or stenosis (Figures 3 and 4). The patient was discharged the following day and instructed to continue dual antiplatelet therapy and aggressive blood pressure and lipid control.

One year later, the patient presented to the hospital with complaints of chest pain and was diagnosed with non-ST elevation myocardial infarction. During cardiac catheterization, he was found to have approximately $75 \%$ in-stent restenosis of the LIMA covered stent that was located in the midsegment of the graft. There continued to be complete resolution of the prior aneurysm. Repair within the stenosed section was performed with a $3.0 \times 20 \mathrm{~mm}$ drug-eluting stent. Postintervention showed no residual stenosis and TIMI-3 blood flow. Ultimately, the patient was discharged home to continue lifelong dual antiplatelet therapy with aggressive blood pressure and lipid control.

\section{Discussion}

Arterial aneurysms usually occur in elderly men, as well as in individuals that have uncontrolled high blood pressure [6]. They are also typically asymptomatic, small, and do not pose a significant health risk [6]. The most common clinical presentation of a symptomatic graft aneurysm is chest pain and hemoptysis [7].

3.1. Epidemiology. A modest number of reported cases of these aneurysms have been documented since 1978, with 51.9 years noted as a mean age of occurrence (Table 1) [8]. Aneurysms and pseudoaneurysms found in the IMA are seldomly seen outside of ailments involving hereditary diseases, infections, iatrogenic causes, vasculitis, persistent atherosclerosis, or physical trauma [9]. It is an increased likelihood that 


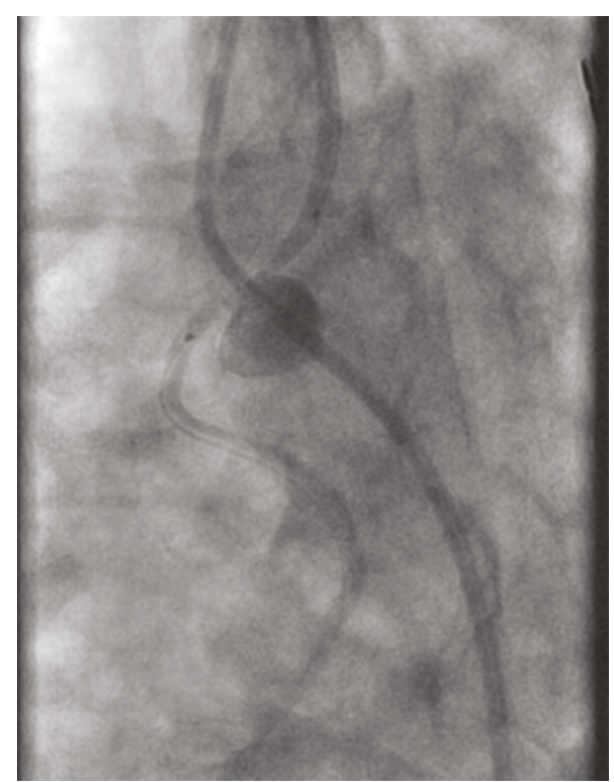

Figure 2: Covered stent placement in the midsegment of a large left internal mammary artery bypass graft aneurysm.

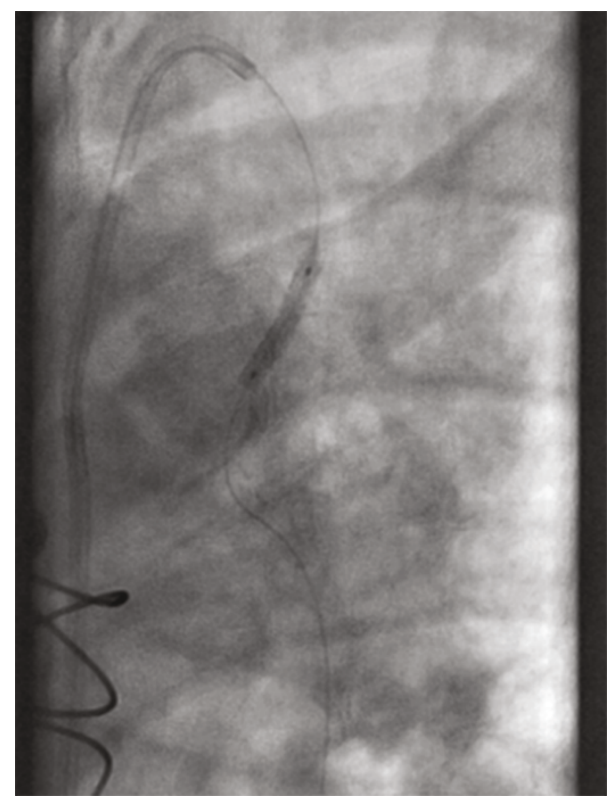

Figure 3: Stent placement within the left internal mammary artery bypass graft.

graft endothelial dysfunction, atherosclerotic changes, and changes in the orientation of the medial smooth muscle have some role in late aneurysm development [9].

IMAs are fundamentally ideal for use during bypass grafting due to a decreased tendency for spasms and reduced development of atherosclerosis [4]. Procedurally, the LIMA is removed from the chest wall and the proximal end remains attached to the subclavian artery, with the distal end newly connected to the LAD distal to the site of occlusion (Figure 5) [4]. One theory suggests that skeletonization of the IMA to preserve sternal blood perfusion in fact deprives



Figure 4: Postintervention angiography showing no residual aneurysm or stenosis in the left internal mammary artery bypass graft.

the grafted artery of possible long-term sustainability because it adversely affects its resistance to atherosclerosis by affecting its original nerve supply, vasa vasorum, and lymphatic drainage [10]. With this theory, it may be a disadvantage to perform grafting with this method in comparison to using pedicled grafting or covered stents in a person with a known history of severe atherosclerosis [10].

3.2. Causes. The IMA's pregrafting location adjacent to the sternum provides increased susceptibility to penetrating injury, blunt chest trauma, and iatrogenic injury [6]. A few case reports have described the existence of these aneurysms following pacemaker lead placement or a complicated central venous catheter placement [11]. An idiopathic IMA aneurysm without a recent medical history to explain its occurrence is extremely rare [11]. IMA aneurysms can be classified into various groups such as early versus late presentation [12].

Early IMA graft aneurysms may be due to tension at the site of anastomosis or may be related to an implanted graft infection. Other causes include technical errors during anastomosis, intimal hyperplasia, direct trauma from a median sternotomy, or from atheroma formation [11]. It is likely that the patient in this case developed a LIMA aneurysm in a short time following his CABG due to iatrogenic trauma related to grafting. Late graft aneurysms are likely secondary to progressive or accelerated atherosclerotic degeneration that is due to patient medication noncompliance or resistance to treatment. IMA grafts, however, are usually resistant to the formation of atherosclerosis, which contributes to longer patency rates when compared to subclavian vein grafts [4].

3.3. Diagnosis. Aneurysms that are symptomatic pose a challenge diagnostically because they may present as congestive heart failure or acute coronary syndrome [12]. Having an 
TABLE 1: Previously reported artery aneurysms [8].

\begin{tabular}{|c|c|c|c|c|}
\hline Study (year) & Location & Etiology & Management & Discovery \\
\hline $\begin{array}{l}\text { Otter and Stam [5] } \\
\text { (1978) }\end{array}$ & $\begin{array}{l}\text { Left internal } \\
\text { mammary artery }\end{array}$ & Unknown & Left lateral thoracotomy and ligation & "Coin lesion" on chest X-ray \\
\hline $\begin{array}{l}\text { Chan and } \\
\text { Fermanis [1] } \\
\text { (1995) }\end{array}$ & $\begin{array}{c}\text { Left internal } \\
\text { mammary artery }\end{array}$ & Unknown & Angiographic embolization & Spontaneous hemothorax \\
\hline $\begin{array}{l}\text { Kugai and Chibana } \\
\text { [8] (1999) }\end{array}$ & $\begin{array}{l}\text { Left internal } \\
\text { thoracic artery }\end{array}$ & Atherosclerosis & $\begin{array}{l}\text { Internal thoracic artery } \\
\text { aneurysmectomy and reconstruction }\end{array}$ & "Angina" \\
\hline $\begin{array}{l}\text { Lindblom et al. } \\
\text { [14] (2013) }\end{array}$ & $\begin{array}{l}\text { Left internal } \\
\text { thoracic artery }\end{array}$ & Unknown & Endovascular coiling & $\begin{array}{l}\text { Left-sided shoulder pain } \\
\text { radiating to chest }\end{array}$ \\
\hline $\begin{array}{l}\text { Heyn et al. [15] } \\
\text { (2014) }\end{array}$ & $\begin{array}{l}\text { Left internal } \\
\text { mammary artery }\end{array}$ & $\begin{array}{c}\text { Thrombotic obliterated } \\
\text { aneurysm }\end{array}$ & Open surgical resection & Preoperative chest X-ray \\
\hline Present case (2018) & $\begin{array}{l}\text { Left internal } \\
\text { mammary artery }\end{array}$ & $\begin{array}{l}\text { Iatrogenic trauma during } \\
\text { bypass grafting }\end{array}$ & Covered stent & $\begin{array}{l}\text { Incidental finding during } \\
\text { cardiac catheterization }\end{array}$ \\
\hline
\end{tabular}

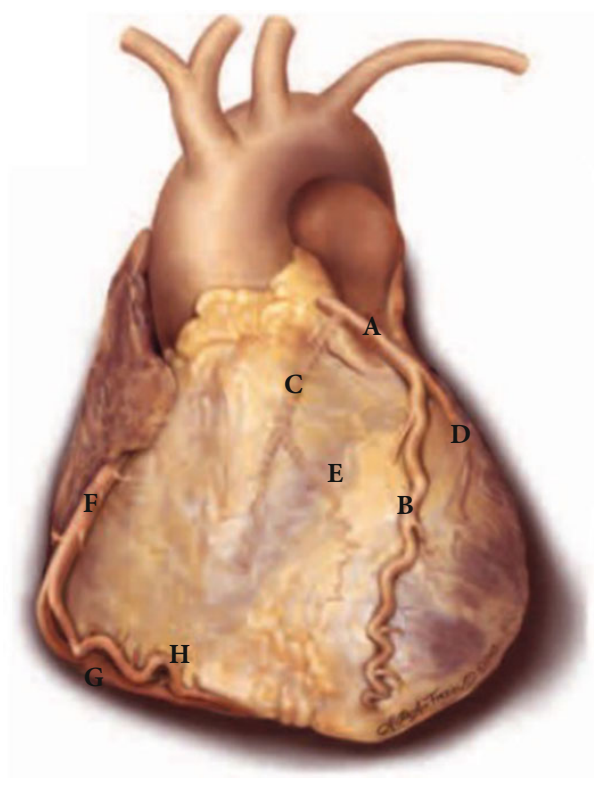

(a)

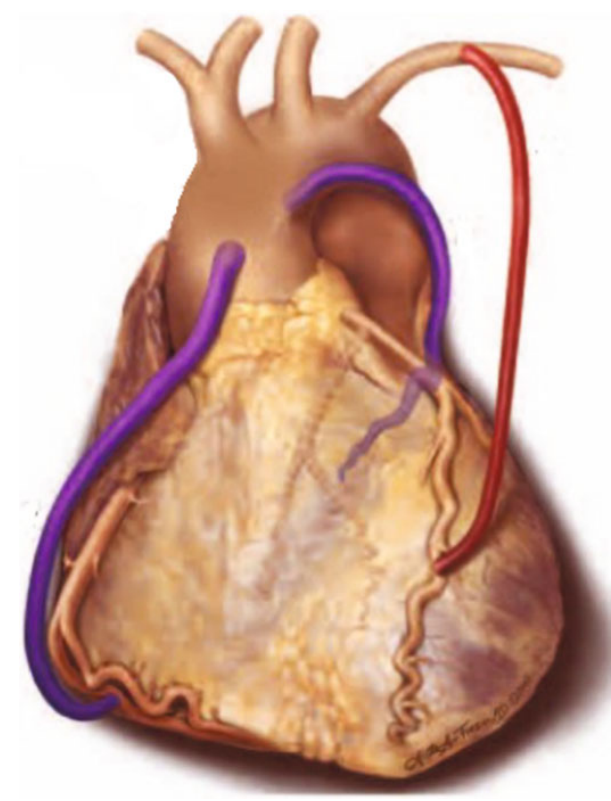

(b)

FIGURE 5: (a) Normal anatomy of the coronary arteries: (A) left coronary artery, (B) left anterior descending artery, (C) left circumflex artery, (D) diagonal artery, (E) obtuse marginal branch of the left circumflex artery, (F) right coronary artery, (G) posterior descending artery, and $(\mathrm{H})$ acute marginal branch of the right coronary artery. (b) Left internal mammary artery graft from its origin at the subclavian artery proximally and grafted to the left anterior descending artery distally (in red) [4]. This figure is reproduced from Frazier, A., Qureshi, F., Read, K. M., Gilkeson, R. C., Poston, R. S., \& White, C. S. (2005). Coronary Artery Bypass Grafts: Assessment with Multidetector CT in the Early and Late Postoperative Settings. Radiographics, 25(4), 881-896 (under the Creative Commons Attribution License/public domain).

asymptomatic clinical course, no current guidelines for screening, initial presentation of sudden death from rupture, and incidental finding on diagnostic imaging are some reasons that may contribute to the underdiagnosing of this phenomena [2]. Late presentation is also another reason that these aneurysms are likely to be missed. About $90 \%$ of saphenous vein graph aneurysms manifest greater than five years following bypass surgery, with a mean time of about 13 years before final diagnosis [2].

Reportedly, about $50 \%$ of aneurysm cases can be observed incidentally on chest X-ray and will show a hilar or mediastinal mass [4]. In one report, a pulmonologist doc- umented that a patient that was referred to him for an evaluation of a coin lesion noted on imaging in fact was discovered to have an IMA aneurysm [5]. Other diagnostic modalities include echocardiogram, contrast-enhanced thoracic CT scan, CT angiography, and MRI angiography. The definitive imaging test for confirming an aneurysm is coronary artery CT angiography, as it will show the extent of the aneurysm, local mass effect, and will note a change in the vasculature [7]. Findings may be obscured if there is a mural thrombus involved [13]. In coming years, increased early identification of bypass aneurysms will likely be reported due to continuous improvement of long-term outcomes following coronary 


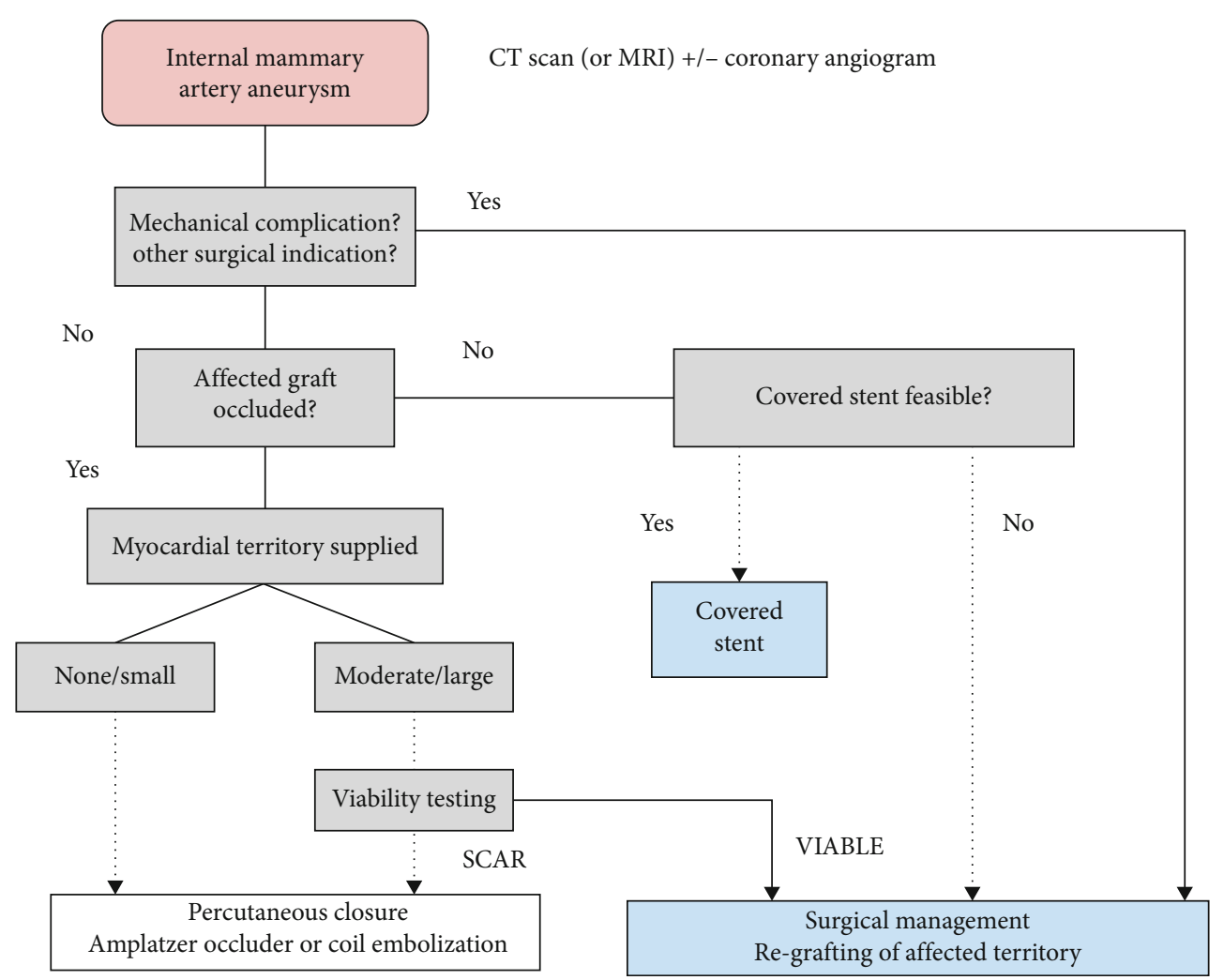

FIGURE 6: Suggested algorithm for assessment and treatment of an internal mammary artery aneurysm. CT: computed tomography; MRI: magnetic resonance imaging [7].

artery bypass grafting and due to the increasing sophistication of diagnostic investigations [6].

3.4. Treatment. When a patient is diagnosed with an IMA aneurysm, it is highly recommended to begin prompt therapy to prevent life-threatening complications [11]. About $20 \%$ of patients with saphenous vein aneurysms are treated with conservative management comprising of risk factor modification, secondary prevention, and surveillance [2]. Reducing the risk of mass effect, thromboembolism, rupture, and myocardial ischemia are the main goals of treatment [2]. Options for invasive treatment are surgical repair or percutaneous approaches, which include vascular coil embolization, covered stenting, and vascular occlusion (Figure 6) [7]. Covered stenting has a major advantage in that it protects distal flow patency, unlike other percutaneous options. Surgery should be immediately considered if the patient has a high risk of rupture or if the aneurysm is causing compressive complications [2]. Surgical techniques include ligation of the aneurysm or resection of the aneurysm with or without bypass revascularization [2]. At a minimum, patients should receive a complete cardiac evaluation by an interventional cardiologist and a cardiac surgeon [7].

\section{Conclusion}

IMA aneurysms represent a very rare complication following a CABG. It has a high potential for morbidity and mortality, and the risk of complications will likely rise with the size of the aneurysm [2]. Increased attention to and awareness of this dis- ease process will hopefully facilitate earlier diagnosis. Coronary CT angiography is the most definitive test for not only confirming but also discovering possible complications stemming from the aneurysm [13]. With continued improvement in long-term outcomes following bypass grafting since the 1960s, and with an increase in the aging population undergoing bypass surgery, it is likely that the incidence of documented IMA aneurysms or pseudoaneurysms will grow [7, 12]. Endovascular treatment is especially effective for the severely ill and elderly population, with the number of successful aneurysm cases treated with this method continuing to rise [6]. Following an extensive literature review, and to the best of our knowledge, this is the first documented case of a true aneurysm found within a LIMA bypass graft. It is our hope that this review will further assist clinicians in increasing accurate evaluation and management of this rare disease process.

\section{Data Availability}

Data is available upon request to the corresponding author.

\section{Conflicts of Interest}

The authors declare that they have no conflicts of interest.

\section{Authors' Contributions}

Osayi Lawani's contributions consisted of conceptualization, data curation, investigation, creation of the original draft, 
review and editing, and visualization. Rick Ganim's contributions consisted of supervision, conceptualization, methodology, validation, and review and editing of the original draft. Jiries Ganim contributed in data curation, investigation, and review and editing of the original draft.

\section{Acknowledgments}

The authors would like to especially thank John Washington IV, Laura Tinnell, Misty Jennings, and Richard Shannon.

\section{References}

[1] L. W. Chan and G. G. Fermanis, "Spontaneous hemothorax caused by an internal mammary artery aneurysm," The Australian and New Zealand Journal of Surgery, vol. 66, no. 5, pp. 332-333, 1996.

[2] J. T. Connell, "Saphenovenous graft aneurysm: a rare complication of CABG," Case Reports in Cardiology, E. Ercan, Ed., vol. 2017, Article ID 8101489, 2017.

[3] S. J. Head, T. M. Kieser, V. Falk, H. A. Huysmans, and A. P. Kappetein, "Coronary artery bypass grafting: part 1-the evolution over the first 50 years," European Heart Journal, vol. 34, no. 37, pp. 2862-2872, 2013.

[4] A. Frazier, F. Qureshi, K. M. Read, R. C. Gilkeson, R. S. Poston, and C. S. White, "Coronary artery bypass grafts: assessment with multidetector CT in the early and late postoperative settings," Radiographics, vol. 25, no. 4, pp. 881-896, 2005.

[5] G. D. Otter and J. Stam, "Aneurysm of internal mammary artery," Thorax, vol. 33, no. 4, pp. 526-527, 1978.

[6] M. Miyazaki, H. Nagamine, H. Hara, H. Sugita, and Y. Kawase, "Successful treatment of a right internal mammary artery aneurysm with thoracoscopic surgery," Journal of Vascular Surgery Cases and Innovative Techniques, vol. 5, no. 3, pp. 269-272, 2019.

[7] D. Ramirez, B. Hibbert, T. Simard et al., "Natural history and management of aortocoronary saphenous vein graft aneurysms," Circulation, vol. 126, no. 18, pp. 2248-2256, 2012.

[8] T. Kugai and M. Chibana, "Non-trauma-induced aneurysm of the left internal thoracic artery with ischemic heart disease-a case report and review of the literature," Japan Journal of Cardiovascular Surgery, vol. 28, no. 4, pp. 260-263, 1999.

[9] I. K. Klair and J. Palomino, "Giant saphenous vein coronary artery bypass graft aneurysm," American Journal of Respiratory and Critical Care Medicine, vol. 189, no. 5, pp. 8-9, 2014.

[10] J. Francis, "Skeletonized internal mammary graft vs pedicled graft," in Cardiac Surgery, Kozhikode, Kerala, India, 2018, July 2020, https://johnsonfrancis.org/professional/skeletonizedinternal-mammary-graft-vs-pedicled-graft/.

[11] T. Almerey, R. Paz-Fumagalli, H. Farres, W. A. Oldenburg, and A. G. Hakaim, "Idiopathic internal mammary artery aneurysm in the setting of aberrant right subclavian artery," Journal of Vascular Cases and Innovative Techniques, vol. 3, no. 4, pp. 251-253, 2017.

[12] M. Doyle, D. L. Spizarny, and D. E. Baker, "Saphenous vein graft aneurysm after coronary artery bypass surgery," American Journal of Roentgenology, vol. 168, pp. 747-749, 1996.

[13] C. Burke, S. Shalhub, and B. W. Starnes, "Endovascular repair of an internal mammary artery aneurysm in a patient with SMAD-3 mutation," Journal of Vascular Surgery, vol. 62, no. 2, pp. 486-488, 2015.
[14] R. Lindblom, V. Zemgulis, A. Lilieqvist, and R. Nyman, "Even small aneurysms can bleed: a ruptured small idiopathic aneurysm of the internal thoracic artery," Interactive Cardiovascular and Thoracic Surgery, vol. 17, no. 3, pp. 583-585, 2013.

[15] J. Heyn, H. Zimmermann, A. Klose, B. Luchting, C. Hinske, and M. S. Azandaryani, "Idiopathic internal mammary artery aneurysm," Journal of Surgical Case Reports, vol. 12, pp. 1-3, 2014. 